\title{
Sound equalization in a large region of a rectangular enclosure
}

\author{
Sarris, John C.; Jacobsen, Finn; Cambourakis, Georg E.
}

Published in:

Acoustical Society of America. Journal

Link to article, DOI:

$10.1121 / 1.1819500$

Publication date:

2004

Document Version

Publisher's PDF, also known as Version of record

Link back to DTU Orbit

Citation (APA):

Sarris, J. C., Jacobsen, F., \& Cambourakis, G. E. (2004). Sound equalization in a large region of a rectangular enclosure. Acoustical Society of America. Journal, 116, 3271-3274. https://doi.org/10.1121/1.1819500

\section{General rights}

Copyright and moral rights for the publications made accessible in the public portal are retained by the authors and/or other copyright owners and it is a condition of accessing publications that users recognise and abide by the legal requirements associated with these rights.

- Users may download and print one copy of any publication from the public portal for the purpose of private study or research.

- You may not further distribute the material or use it for any profit-making activity or commercial gain

- You may freely distribute the URL identifying the publication in the public portal

If you believe that this document breaches copyright please contact us providing details, and we will remove access to the work immediately and investigate your claim. 


\title{
Sound equalization in a large region of a rectangular enclosure (L)
}

\author{
John C. Sarris ${ }^{\text {a) }}$ and Finn Jacobsen ${ }^{\text {b) }}$ \\ Acoustic Technology, Ørsted DTU, Technical University of Denmark, Ørsteds Plads, \\ Building 352, DK-2800 Kgs. Lyngby, Denmark \\ George E. Cambourakis ${ }^{\mathrm{c})}$ \\ School of Electrical and Computer Engineering, National Technical University of Athens, \\ Heroon Polytechniou 9, 157 73, Athens, Greece
}

(Received 13 February 2004; revised 14 September 2004; accepted 24 September 2004)

\begin{abstract}
The work presented by Santillán [J. Acoust. Soc. Am. 110, 1989-1997 (2001)] about equalization at low frequencies in rectangular enclosures is extended, and topics that remained unaddressed in the original study are treated in this paper. A modification is introduced to the original cost function that leads to a least-squares problem formulation that demonstrates increased robustness, and the multiple-error LMS (Least Mean Square) adaptive algorithm is employed to approximate the coefficients of the equalization filters to their optimum values. Other issues studied in this paper include the dependence of the limits of the zone of equalization on factors such as the damping constant of the modes of the enclosure, the number of sources, and the driving frequency. (C) 2004 Acoustical Society of America. [DOI: 10.1121/1.1819500]
\end{abstract}

PACS numbers: 43.55.Br, 43.60.Pt, 43.60.Mn [NX]

Pages: $3271-3274$

\section{INTRODUCTION}

The purpose of equalization in room acoustics is to compensate for the undesired modification that a particular enclosure introduces to signals as, for example, audio or speech. Traditional multichannel methods introduce digital filters to preprocess the input signal before it is fed to a set of loudspeakers so that the spectral coloration and the reverberation tail associated with the transmission path are removed and the obtainable signals at a set of receiving points are the best approximations to a set of desired signals. ${ }^{1}$ Such techniques exhibit a high performance exactly at the receiving points, but the zone where equalization can be performed is limited to small areas in the vicinity of the points. ${ }^{2}$

The problems that traditional equalization methods face were overcome in Santillán's approach. ${ }^{3}$ In this work a plane wave moving along a rectangular enclosure was generated in order to equalize the sound field at low frequencies in a continuous three-dimensional region that occupied almost the complete volume of the enclosure. Theoretical results showed that the generation of a traveling plane wave is possible within a rectangular enclosure provided that sets of sources are arranged in the two walls that are perpendicular to the direction of propagation. The traditional least-squares approximation has been employed to estimate the coefficients of FIR filters for the equalization of broadband signals. $^{3}$

However, some issues remained unaddressed in the original study, and thus the purpose of this paper is to extend

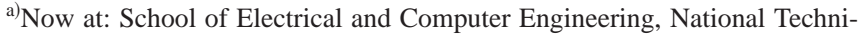
cal University of Athens, Heroon Polytechniou 9, 157 73, Athens, Greece. Electronic mail: jsarris@central.ntua.gr

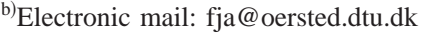

${ }^{c}$ Electronic mail: gcamb@cs.ntua.gr
}

the previous work and discuss some matters more thoroughly on the basis of simulation and experimental results.

\section{EQUALIZATION OF HARMONIC SOUND FIELDS}

\section{A. Problem formulation and optimum solution}

In the work by Santillán ${ }^{3}$ the optimum strengths of the sources for the equalization of a harmonic sound field by simulating a propagating plane wave are obtained according to the multiple point method ${ }^{4}$ by minimizing the meansquared error at a number of receivers distributed in the zone of equalization. However, under certain conditions this leastsquares formulation fails to provide a solution or provides one that lacks robustness.

A more robust solution for the optimum strengths of the sources can be obtained by including a term proportional to the effort of the sound sources in the cost function. Minimization of this modified cost function is guaranteed to provide a solution in all cases, although it increases the minimum value of the cost function and thus in general makes the solution slightly less perfect. Furthermore, since in practice the strengths of the sources are approximated to their optimal values by using an adaptive algorithm such as, for example, the multiple-error LMS algorithm, ${ }^{5}$ this modification ensures convergence. In the following section simulation results are presented to support this.

\section{B. Simulation results}

\section{An ill-conditioned system}

The purpose of the first simulation is to demonstrate the different performance of the cost function used by Santillán ${ }^{3}$ and the modified cost function proposed in this study when the system is ill-conditioned. The enclosure considered in the simulation is rectangular, with dimensions $L_{x}=2.7 \mathrm{~m}, L_{y}$ 
$=5 \mathrm{~m}$, and $L_{z}=2.5 \mathrm{~m}$. All modes with a natural frequency up to $1 \mathrm{kHz}$ are used to simulate the sound pressure. Thirtytwo sources and 36 receivers are considered placed as described by Santillán. ${ }^{3}$ The driving frequency is $200 \mathrm{~Hz}$ and a damping constant with value equal to 0.01 for all the modes is assumed.

The transfer function matrix that results from the arrangement of sources and receivers described above is rank deficient, and thus the least-squares formulation proposed by Santillán $^{3}$ does not have a numerically stable solution. However, the cost function with effort weighting leads to a formulation that provides a stable solution. With the LMS adaptive formulation of the cost function without effort weighting there are some modes with infinite time constants, since the transfer function matrix is rank deficient. The inclusion of the effort weighting in the definition of the cost function compensates for the rank deficiency and ensures that there are no such modes.

\section{Limits of the zone of equalization}

According to Santillán's study ${ }^{3}$ the zone of equalization, defined as the continuous three-dimensional region of the enclosure where the deviations of the sound pressure from the desired value are within $\pm 3 \mathrm{~dB}$, is constantly limited to be $0.6 \mathrm{~m}$ away from the two parallel walls where the sources are placed. This limit is a conservative estimate for most practical cases, and thus the dependence of this restriction on factors like the damping constant of the modes of the enclosure, the number of sources, and the driving frequency calls for further examination.

From simulation results it can be concluded that the limits of the zone of equalization depend on all three factors, i.e., the number of sources, the driving frequency, and the damping constant of the modes of the enclosure. In practical cases where a finite number of sources is used the zone of equalization will be limited with respect to the walls where the sources are placed, and the distance of the limits from these walls will be proportional to the driving frequency and inversely proportional to the number of the sources. Furthermore, the results of simulations show that in general damping tends to limit the zone of equalization further.

\section{EQUALIZATION OF BROADBAND SOUND FIELDS}

\section{A. Problem formulation and optimum solution}

As shown by Santillán, it is possible to equalize a broadband signal in a large region in a rectangular room by generating a traveling plane wave. ${ }^{3}$ In his study, equalization of a broadband signal was performed by FIR equalization filters whose coefficients were determined by minimization of the expectation of the sum of squared errors at a number of receivers distributed in the zone of equalization. ${ }^{6}$ However, as in the case of harmonic sound-field equalization, this least-squares formulation may fail to provide a solution for certain arrangements of sources and receivers.

The introduction of a term proportional to the sum of the squared equalization coefficients to the cost function results in a least-squares formulation of increased robustness and, as in the case of the adaptive implementation for the equaliza- tion of a single-tone sound field, the convergence stability of the LMS formulation of the modified cost function is guaranteed.

\section{B. Experimental results}

Experimental results have been obtained in a rectangular enclosure constructed of 22-mm MDF fiberboard with internal dimensions $L_{x}=1.2 \mathrm{~m}, L_{y}=2 \mathrm{~m}$, and $L_{z}=0.2 \mathrm{~m}$. For simplicity the enclosure was much smaller in one dimension than in the other two, so that the resulting sound field is two-dimensional in the frequency range considered. Two holes were made on each side perpendicular to the $y$ axis (the direction of the traveling wave), and two 5-in. loudspeakers were placed in a line at $z=0.1 \mathrm{~m}$ with their respective centers positioned at $x=0.3 \mathrm{~m}$ and $x=0.9 \mathrm{~m}$ in each line. Thus, a total of four sources was used. According to the specification provided by the manufacturer (Vifa-Speak) the loudspeakers have a flat frequency response in the frequency range from about $140 \mathrm{~Hz}$ up to approximately $2 \mathrm{kHz}$. Holes were also made in one of the large sides of the enclosure, and five 9.7-mm electret microphones were distributed within the enclosure in three lines parallel to the $x$ axis, with two microphones in each of the two first lines and a single microphone in the last one. The number of microphones was limited by the memory of the available DSP board. The first line of microphones was positioned at $y=0.6 \mathrm{~m}$, and the distance between adjacent microphones was 0.43 and $0.8 \mathrm{~m}$ in the $y$ and $x$ direction, respectively. Since the sound field in the frequency range of interest should be practically independent of the $z$ direction, all the microphones were placed in a single plane at $z=0 \mathrm{~m}$. In order to measure the sound pressure at various points in the enclosure, 19 holes were evenly made in one side of the enclosure along the $y$ direction. Thus, by traversing a 9.7-mm electret microphone to 13 evenly spaced positions along the $x$ axis $(x=0$ to $1.2 \mathrm{~m})$ in each of the 19 holes the sound pressure could be measured at 247 positions in the enclosure.

The input signal was random noise generated and bandpass filtered in the frequency range from $100 \mathrm{~Hz}$ up to 300 $\mathrm{Hz}$ by the Brüel \& Kjær PULSE system and additionally low-pass filtered by an analog filter with a cutoff frequency of $310 \mathrm{~Hz}$. The input signal was then amplified, and passed through an A/D converter before it was fed to the DSP board. Each of the signals from the microphones was amplified by amplifiers that were adjusted so that each channel had the same sensitivity, passed through an A/D converter, and then transferred to the DSP board. The signals to drive the loudspeakers were generated in the DSP board, passed through a D/A converter, amplified, and then fed to the loudspeakers. Analog antialising filters with a cutoff frequency of $400 \mathrm{~Hz}$ were also used to low-pass filter the amplified signals from the microphones, and analog reconstruction filters with a cutoff frequency of $400 \mathrm{~Hz}$ were used to low-pass filter the output signals of the DSP before they were fed to the amplifiers and loudspeakers. The card with the A/D converter, the card with the D/A converter, and the DSP board were housed in a Pentium 233-MHz PC. The DSP board was manufactured by Loughborough Sound Images Ltd. and had implemented a Motorola DSP 96002 processor with a 33.3-MHz 


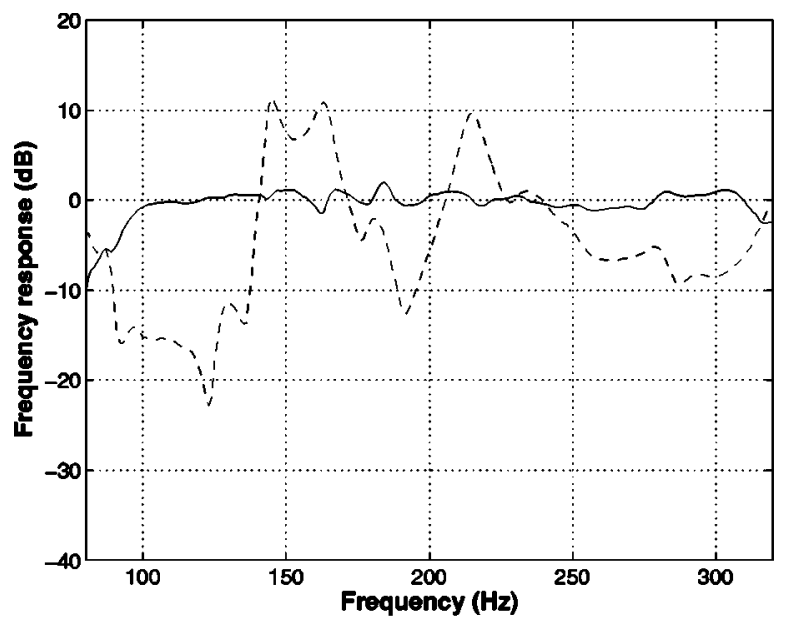

(a)

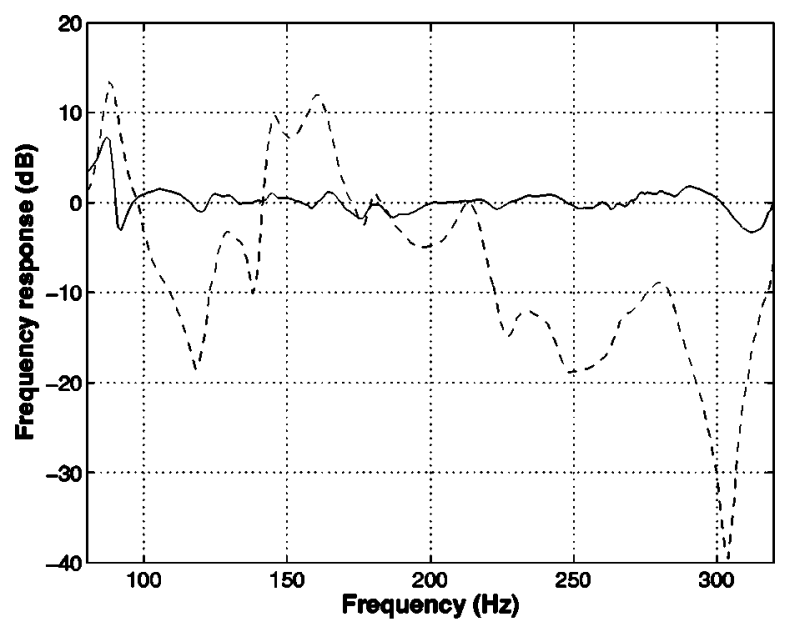

(c)

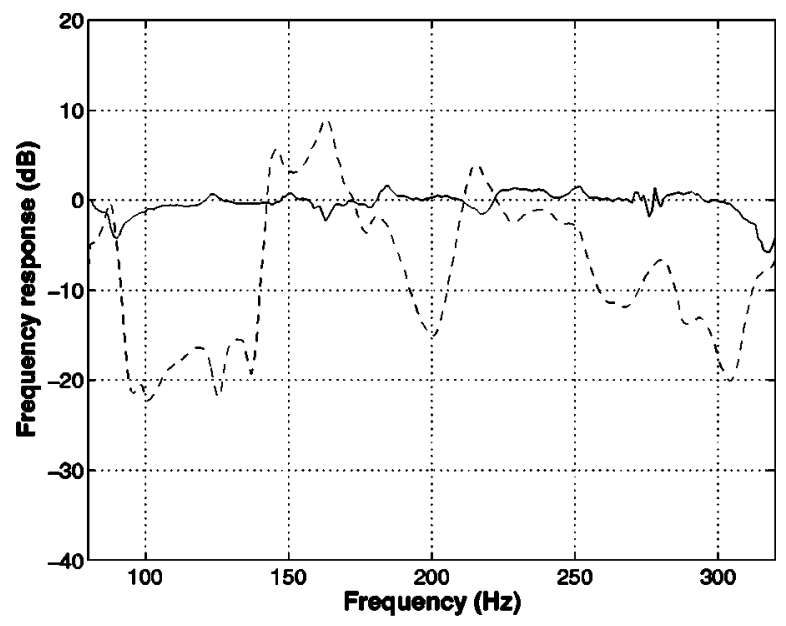

(b)

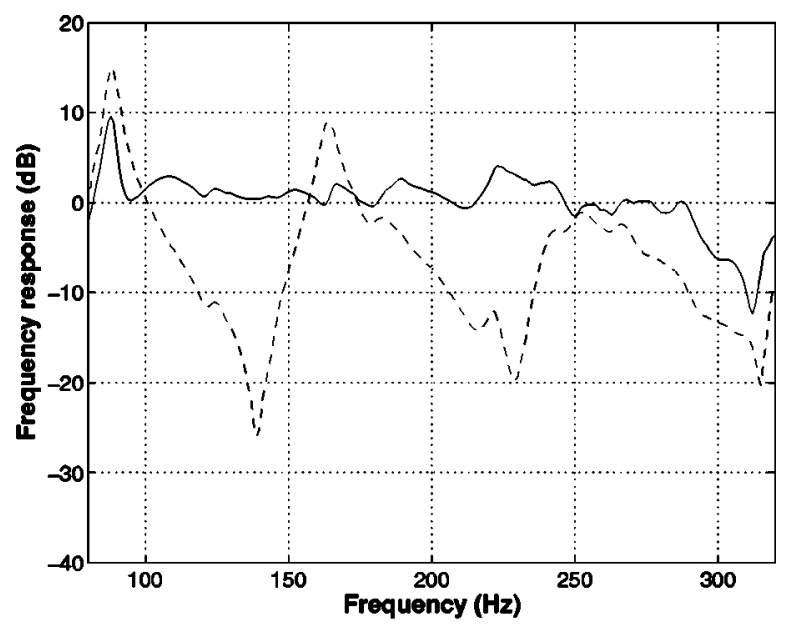

(d)

FIG. 1. Frequency responses before (- -) and after (-) equalization at the points (a) $(0.2,1.03,0.1) \mathrm{m} ;(\mathrm{b})(0.4,1.1,0.1) \mathrm{m} ;(\mathrm{c})(1.0,1.6,0.1) \mathrm{m}$; and (d) $(0.6,0,0.1) \mathrm{m}$ corresponding to the location of a microphone used in the equalization, a point within the zone of equalization, a point just outside the zone of equalization, and a point far from the zone of equalization.

clock frequency. The A/D converter was a 32-channel analog acquisition card, and the D/A converter was a 16-channel analog output card, both manufactured by Loughborough Sound Images Ltd.

The impulse responses of the transmission paths between the loudspeakers and the microphones were represented by FIR filters with 150 coefficients, estimated by the multiple-error LMS algorithm implemented as an identification algorithm. Once the identification was completed the coefficients of the identification FIR filters were kept fixed and the equalization process began. The equalization filters were FIR filters with 60 coefficients. The sampling frequency was $1600 \mathrm{~Hz}$, and the propagating plane wave was generated by applying delays of 20 samples to obtain the desired signal for the two receivers in the first line and additional delays of two samples for the receivers in the following lines. These two sample delays correspond to the time that the propagating wave requires to travel from each line of receivers to the next.

The results obtained for the equalization of the broadband sound field by the multiple-error LMS implementation of the modified cost function are illustrated in Fig. 1 at four positions in the enclosure, along with the unequalized frequency responses obtained by exciting the enclosure with the loudspeaker placed at $(0.3,0,0.1) \mathrm{m}$. The frequency responses at the four positions correspond to the location of a microphone used in the equalization, a point within the zone of equalization, a point just outside the zone of equalization, and a point far from the zone of equalization. It can be seen that the frequency responses are improved at all positions after the equalization. The peaks and dips of the original frequency responses are removed and practically flat responses are obtained in the frequency range of interest. Even at the point far from the zone of equalization a reasonably flat response is obtained after the equalization almost in the entire frequency range of concern. Similar results have been obtained at other positions in the enclosure. The spatial distribution of the sound pressure in the $200-\mathrm{Hz}$, one-thirdoctave band before and after the broadband sound field equalization is shown in Fig. 2. The sound pressure before the equalization was obtained by exciting the enclosure with the source placed at $(0.3,0,0.1) \mathrm{m}$. It can be seen that the equalization removed the spatial fluctuations almost in the entire volume of the enclosure, and thus considerably im- 


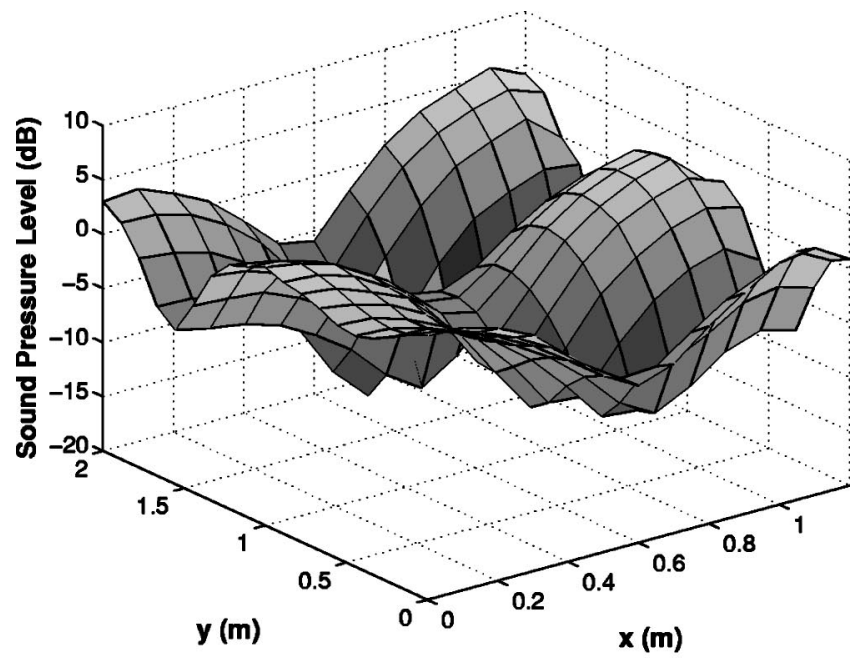

(a)

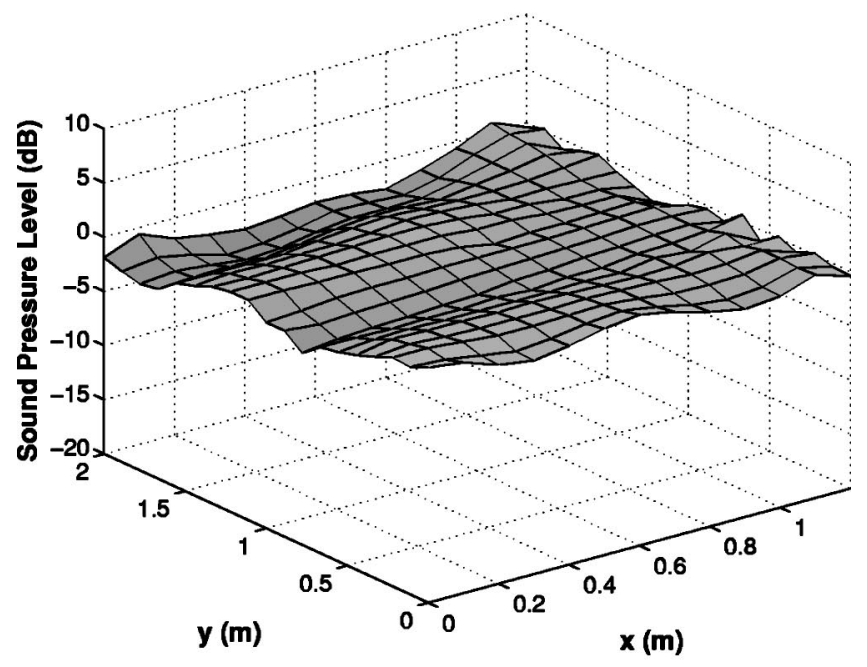

(b)

FIG. 2. Sound-pressure level in the $200-\mathrm{Hz}$ one-third-octave band in the experimental enclosure (a) before, and (b) after equalization with random noise as the input signal. The sound pressure before the equalization is obtained by exciting the enclosure with the source placed at $(0.3,0,0.1) \mathrm{m}$.

proved the spatial distribution of the sound field.

To examine the robustness of the adaptive implementation of the modified cost function in the presence of modeling errors, three additional holes were made at the points $(0.2,0.63,0) \mathrm{m},(0.23,1.46,0) \mathrm{m}$, and $(1,1.49,0) \mathrm{m}$, and the three microphones originally placed at $(0.2,0.6,0) \mathrm{m},(0.2$, $1.46,0) \mathrm{m}$, and $(1,1.46,0) \mathrm{m}$ were moved at these positions after the identification and before the equalization process. Two of these new positions were shifted from the original

positions along the direction of the wave propagation, while the last one was shifted in the $x$ direction. It should be noted that this type of error corresponds to the worst case of model uncertainties. ${ }^{7}$ By proper adjustment the multiple-error LMS implementation of the modified cost function was found to be stable, and the results obtained for the equalization of the broadband sound field were similar to the ones presented above.

\section{CONCLUSIONS}

As shown theoretically by Santillán, ${ }^{3}$ the most efficient way to equalize the sound pressure in a large region in a room is to generate a plane propagating wave. This work supplements Santillán's simulation study; the method has been examined experimentally, and several issues that remained unaddressed in the original study have been treated in this paper. The modification that is introduced to the original cost function leads to a least-squares problem formulation that is guaranteed to provide a solution, and the multiple-error LMS adaptive formulation that is based on the modified cost function has been shown to alleviate problems concerning convergence stability and convergence speed that can occur in the adaptive implementation of the original cost function. Also, it has been shown that in the practical case where a finite number of sources is used the zone of equalization will be limited with respect to the walls where the sources are placed, and the distance of the limits from these walls will be proportional to the driving frequency and inversely proportional to the number of the sources.

\section{ACKNOWLEDGMENTS}

The authors would like to thank A. O. Santillán for making part of the simulation code available and for many helpful discussions. The work of the first author was supported by a Marie Curie Fellowship under the program of European Doctorate in Sound and Vibration Studies.

\footnotetext{
${ }^{1}$ M. Miyoshi and Y. Kaneda, "Inverse filtering of room acoustics," IEEE Trans. Acoust., Speech, Signal Process. 36, 145-152 (1988).

${ }^{2}$ P. A. Nelson, F. O. Bustamante, and H. Hamada, "Inverse filter design and equalization zones in multichannel sound reproduction," IEEE Trans. Speech Audio Process. 3, 185-192 (1995).

${ }^{3}$ A. O. Santillán, "Spatially extended sound equalization in rectangular rooms," J. Acoust. Soc. Am. 110, 1989-1997 (2001).

${ }^{4}$ F. Asano and D. C. Swanson, "Sound equalization in enclosures using modal reconstruction," J. Acoust. Soc. Am. 98, 2062-2069 (1995).

${ }^{5}$ S. J. Elliott, I. M. Stothers, and P. A. Nelson, "A multiple error LMS algorithm and its application to the active control of sound and vibration," IEEE Trans. Acoust., Speech, Signal Process. 35, 1423-1434 (1987).

${ }^{6}$ S. J. Elliott and P. A. Nelson, "Multiple-point equalization in a room using adaptive digital filters," J. Audio Eng. Soc. 37, 899-907 (1989).

${ }^{7}$ A. Omoto and S. J. Elliott, "The effect of structured uncertainty in the acoustic plant on multichannel feedforward control systems," IEEE Trans. Speech Audio Process. 7, 204-212 (1999).
} 